\title{
Electronic access to health information and/or knowledge by health professionals to improve practice and patient care (Protocol)
}

McGowan JL, McAuley LM, Dawes M, Grad R, Hannes K, Judd M, Labrecque M, Leduc Y, Pluye P, Robinson V, Wells GA, Tugwell P

McGowan JL, McAuley LM, Dawes M, Grad R, Hannes K, Judd M, Labrecque M, Leduc Y, Pluye P, Robinson V, Wells GA, Tugwell P. Electronic access to health information and/or knowledge by health professionals to improve practice and patient care.

Cochrane Database of Systematic Reviews 2004, Issue 2. Art. No.: CD004749. DOI: 10.1002/14651858.CD004749.

www.cochranelibrary.com 
TABLE OF CONTENTS

HEADER 1

ABSTRACT

BACKGROUND

OBJECTIVES

METHODS

REFERENCES

WHAT'S NEW

HISTORY

CONTRIBUTIONS OF AUTHORS

DECLARATIONS OF INTEREST

SOURCES OF SUPPORT

INDEX TERMS 
[Intervention Protocol]

\section{Electronic access to health information and/or knowledge by health professionals to improve practice and patient care}

Jessie L McGowan 1 , Laura M McAuley², Martin Dawes ${ }^{3}$, Roland Grad 3 , Karin Hannes ${ }^{4}$, Maria Judd 5 , Michel Labrecque ${ }^{6}$, Yvan Leduc 7 , Pierre Pluye $^{8}$, Vanessa Robinson ${ }^{9}$, George A Wells ${ }^{10}$, Peter Tugwell11

1. Institute of Population Health/Ottawa Health Research Institute, University of Ottawa, Ottawa, Canada. 2Institute of Population Health, University of Ottawa, Ottawa, Canada. ${ }^{3}$ Family Medicine, McGill University, Montreal, Canada. ${ }^{4}$ Belgian Centre for EvidenceBased Medicine and Joanna Briggs Institute Australia, Leuven, Belgium. ${ }^{5}$ Research Use/Agente principale de programme, Utilisation de la Recherche, Canadian Health Services Research Foundation/Fondation canadienne de la recherche sur les services de santé, Ottawa, Canada. ${ }^{6}$ Département de médecine familiale, Université Laval - Centre hospitalier universitaire de Québec, Québec, Canada. ${ }^{7}$ Departement de médicine familiale et de médicine d'urgence, Université Laval, Ville de Québec, Canada. ${ }^{8}$ Département de médecine familiale, Université McGill, Montréal, Canada. ${ }^{9}$ Gynaecological Oncology, NHS Lavender Gynaecology, Worcester, UK. ${ }^{10}$ Cardiovascular Research Reference Centre, University of Ottawa Heart Institute, Ottawa, Canada. 11Centre for Global Health, Institute of Population Health, University of Ottawa, Ottawa, Canada

Contact address: Jessie L McGowan, Institute of Population Health/Ottawa Health Research Institute, University of Ottawa, 1 Stewart St. room 206, Ottawa, Ontario, K1N 6N5, Canada.jmcgowan@uottawa.ca.

Editorial group: Cochrane Effective Practice and Organisation of Care Group.

Publication status and date: Unchanged, published in Issue 2, 2009.

Citation: McGowan JL, McAuley LM, Dawes M, Grad R, Hannes K, Judd M, Labrecque M, Leduc Y, Pluye P, Robinson V, Wells GA, Tugwell P. Electronic access to health information and/or knowledge by health professionals to improve practice and patient care. Cochrane Database of Systematic Reviews 2004, Issue 2. Art. No.: CD004749. DOI: 10.1002/14651858.CD004749.

Copyright @ 2009 The Cochrane Collaboration. Published by John Wiley \& Sons, Ltd.

\section{A B S T R A C T}

This is a protocol for a Cochrane Review (Intervention). The objectives are as follows:

To estimate the effectiveness of providing electronic access to health information to health care providers.

We will consider the following comparisons:

-electronic access to information compared to no electronic access in practice setting

-electronic access to information compared to access to print based materials only

We will consider any objective measure of professional behaviour (i.e., use of resource, specialist referrals) or patient outcomes (e.g., length of hospital stay).

We plan to undertake subgroup analyses considering the potential effect modifiers where possible see "exploring heterogeneity". 


\section{B A C K G R O U N D}

Implicit in the movement towards evidence based practice is the need for access to current best evidence. Historically, health care providers have used a variety of information resources to inform their decision-making. Traditional resources included printed materials, such as textbooks, clinical manuals, journals and drug reference books, association meetings, colleagues and patients (Dawes, 2003).

Advances in electronic technologies have made health information more available. For example, CD-ROMs, world wide web (WWW) and PDA formats are now offering health care professionals medical information electronically and in seconds. The information resources accessed by electronic technologies contain a range of information from primary studies that can be found in journals to synthesized sources that can include electronic books.

The WWW is a convenient way to access different types of electronic health information. It provides a gateway to health information that is available for free or through a subscription. Critically appraised and synthesized information can be found in searchable databases such as The Cochrane Library and the ACP Journal Club. Health care professionals can search for primary research in databases, such as MEDLINE, Embase and CINAHL (Cumulative Index of Allied Health Professionals) and find the full-text of the journal article directly in the electronic journals available on the WWW. Guidelines are also available on the WWW and searchable via clearinghouses (for example, the Canadian Medical Association Infobase and the National Guidelines Clearinghouse). Electronic textbooks and manuals that are updated frequently, such as Clinical Evidence and Evidence-Based On Call are also available. To help health care professionals manage the overwhelming amounts of health information available on the WWW, there are many web sites that provide "one-stop shopping", sites that provide links to medical information resources in one place (such as the UK National electronic Library for Health) or provide links to journals, guidelines, textbooks with one search (such as the TRIP database at www.tripdatabase.com). In addition, health care professionals have access to information specifically written for their patients at numerous Association and Society web sites.

Many health care professionals have ready access to the Internet. The Canadian Medical Association 2002 Physician Resource Questionnaire reported that approximately $57 \%$ of physicians in Canada have access to the Internet at their office or clinical practice (CMA, 2002). In New Zealand $40 \%$ of physicians have access at work (Kerse, 2001). While approximately $84 \%$ of physicians in the UK and $48 \%$ in Norway have access to the Internet either at home or at work (rsom), (Nylenna). Allied health professionals appear to have similar access: $66 \%$ of dietitians, and $88 \%$ of nurses in the UK had Internet access at work (Kirk), (Nursing standard). In a recent survey of Canadian physiotherapists $62 \%$ (95\% Cl: 55 to 68 ) reported they had access to the Internet in their practice (Judd 2004).

But does access to, or attempts to provide electronic access to, health information, as opposed to traditional sources of information, such as textbooks in print, improve provider practice and health outcomes for patients? Furthermore, does the type of resource (such as electronic journals) that health care providers have access to or the platform (such as the WWW) from which the information is accessed influence the impact on provider behaviour or patient health outcomes?
We propose to undertake a systematic review of interventions intended to improve or provide electronic access to health information for health care practitioners to improve provider access to electronic resources for health providers to improve patient care. Access to information can be either passive or active. Passive access requires the user to seek the information, equivalent to consulting a textbook. Active access provides information to the user, for example alerts, reminders, drug interactions. We will consider only passive forms of access in this review.

\section{OB JECTIVES}

To estimate the effectiveness of providing electronic access to health information to health care providers.

We will consider the following comparisons:

-electronic access to information compared to no electronic access in practice setting

-electronic access to information compared to access to print based materials only

We will consider any objective measure of professional behaviour (i.e., use of resource, specialist referrals) or patient outcomes (e.g., length of hospital stay).

We plan to undertake subgroup analyses considering the potential effect modifiers where possible see "exploring heterogeneity".

\section{METHO DS}

\section{Criteria for considering studies for this review}

\section{Types of studies}

Randomised controlled trials (RCTs) including cluster randomised trials, controlled clinical trials (CCT), and interrupted time series analyses (ITS) where there is a clearly defined point in time when the intervention occurred and at least three data points before and three after the intervention. Studies will be included regardless of language of publication or publication status.

\section{Types of participants}

Health care providers, including physicians, nurses and allied health care professions (such as physiotherapists, speech pathologists, social workers, etc.) involved in providing direct patient care. This review will exclude students or persons in undergraduate training programs.

\section{Types of interventions}

The following interventions will be considered: -interventions to provide or increase electronic access to information (such as free access to particular journals or databases).

We will consider studies where access is provided at point of patient care delivery or elsewhere in the workplace (e.g. library, offices). What is being made available for access needs to be described by the studies for both the intervention and control groups.

We will include studies that include a training component provided that there is differential access between groups.

Medical knowledge has been defined as "information about diseases, therapies, interpretation of lab tests, etc, which is potentially applicable to decisions about multiple patients and public health policies" (Wyatt, 2002). We will use the broader term 
"health" to mean any type of medical, nursing or allied health information, and use the term information for information or knowledge.

We define electronic access in this review as using electronic information technologies (such as Internet, CD-ROMs, PDAs) to access health information. The review will include passive access to electronic information, which is equivalent to consulting a textbook. This is distinct from active access to information which includes interventions such as alerts or reminders.

We will not review clinical decision support systems or patientrelated data systems which are used to patient specific advice.

\section{Types of outcome measures}

Any objective or blind measure of professional behaviour (i.e., use of resource, specialist referrals) or patient outcomes (e.g., length of hospital stay). Measures of health practitioners' knowledge, attitudes or satisfactions will be collected as secondary outcomes. However, studies reporting only knowledge, attitudes and satisfactions will not be sought or included.

\section{Search methods for identification of studies}

The following electronic databases will be searched:

(a) The EPOC Register (and the database of studies awaiting assessment). See SPECIALISED REGISTER under GROUP DETAILS.

(b) Cochrane Central Register of Controlled Trials (CENTRAL)

(c) MEDLINE, Embase, CINAHL, LISA (Library and Information Science Abstracts), Library Literature \& Information Sciences, Virtual Health Library (BIREME), WHO databases and CAB Health

\section{Other sources:}

(a) Hand searching of relevant conference proceedings which have not already been hand searched on behalf of the Cochrane Collaboration.

(b) Hand searching of selected specialized journal and books in the field of library science and medical informatics.

(c) Reference lists of all papers and relevant reviews identified and selected review of reference lists from cited papers (through Web of Knoweldge).

(d) Authors of relevant papers will be contacted regarding any further published or unpublished work.

(e) Organizations (such as aid agencies, access providers) working with developing countries will be contacted regarding relevant studies of which they may be aware.

Search strategies for electronic databases will be developed using the methodological component of the EPOC search strategy combined with selected MeSH terms and free text terms. The search strategy will be translated into the other databases using the appropriate controlled vocabulary as applicable.

1 exp online systems/

2 databases, bibliographic/ or databases, factual/

3 ((electronic or online or computerized) adj2 (access or retrieval or technolog\$)).tw.

4 exp compact disks/ or cd-rom/

5 Internet/

6 (internet or CD-ROM or cd rom or compact disk\$).tw.

7 (www or world wide web).tw.

8 (pda or personal digital assisstant\$ or handheld or hand held).tw. 9 or $/ 1-8$

10 exp manuals/ or exp reference books/ or textbooks/ or periodicals/
11 (textbook\$ or book\$ or journal\$ or periodical\$ or manual or manuals).tw.

12 exp Guidelines/

13 Information Dissemination/

14 or/10-13

159 and 14

16 databases, bibliographic/

17 (medline or pubmed or gratefulmed or embase or cinahl or cochrane or clinical evidence or mdconsult or inforetrieval).tw.

18 exp "information storage and retrieval"/ or medlars/

19 or/16-18

20 access $\$ . t w$.

2119 and 20

2219 and 15

Terms used to identify studies in MEDLINE

\section{Data collection and analysis}

\section{Screening}

Titles and abstracts will be screened for inclusion independently by two reviewers. Any disagreement will be resolved by discussion between the reviewers and arbitrator(s) as necessary. Full text copies of all papers that are potentially relevant will be retrieved.

\section{Data Abstraction}

Two reviewers will undertake data abstraction independently using a tailored data collection checklist based on the generic EPOC data collection checklist. Any disagreement will be resolved by discussion. An arbitrator will be involved where necessary.

\section{Quality}

The methodological quality of all included studies will be assessed by two reviewers independently using criteria described in the EPOC module (http://www.epoc.uottawa.ca) see ADDITIONAL INFORMATION, ASSESSMENT OF METHODOLOGICAL QUALITY under GROUP DETAILS). Any discrepancies will be resolved by discussion and/or involvement of an arbitrator.

\section{Reporting}

Outcomes will be reported in natural units. Where baseline results are available from RCT, and CCT designs, pre-intervention and post-intervention means or proportions will be reported for both study and control groups and the unadjusted and adjusted (for any baseline imbalance) absolute change from baseline will be calculated with $95 \%$ confidence limits. Dichotomous outcome measures will be presented as both risk differences and relative risk reductions.

For ITS studies we will report both the change in the level of outcome immediately after the introduction of the intervention and the change in the slopes of the regression lines before and after the introduction of the intervention.

\section{Analytical Approach}

\section{Primary analyses:}

Primary analyses will be based upon consideration of dichotomous outcome measures. Where studies report more than one measure for each endpoint, the primary measure (as defined by the study) will be abstracted. Where there is no clear primary measure, we will calculate a median from all available measures. 
For comparisons of RCT and CCT, designs we will report (separately for each study design):

"Median effect size across included studies

"Inter-quartile ranges of effect sizes across included studies

"Range of effect sizes across included studies

Methods for reanalysis of RCT, CCT designs with potential unit of analysis errors:

Unit of analysis errors can occur when the unit of allocation and the unit of analysis differ. For example, where health care providers are randomized and individual patient outcomes are reported without accounting for the clustering of patients during the analysis, significance and precision may be overstated. We will attempt to re-analyse studies with potential unit of analysis errors and will indicate where this was done. Where this is not possible, we will report only the point estimates.

Methods for reanalysis of ITS comparisons with inappropriate analysis:

Time series regression will be used to re-analyse each comparison where possible (Ramsey).

\section{Secondary analyses:}

Continuous outcomes will be used primarily to help with interpretation of the findings. Comparisons of continuous outcomes with dichotomous outcomes will be made in an effort to describe the data.

\section{Exploring heterogeneity:}

We will prepare tables and bubble plots comparing effect sizes of studies grouped according to potential effect modifiers. A bubble plot graphically presents the relationship between the outcome of each study and a given effect modifier with the use of regression lines. Each study is represented by a bubble, the size of the bubble represents a study characteristic, often the size or quality of the study.

\section{The following effect modifiers will be examined:}

Provider: years in practice, type of health care provider (e.g. physician, nurse, physiotherapist etc)

Intervention: method of access (high speed access, dial-up etc), type of access (tailored versus generic access); type of information (databases, e-journals etc); intensity of training (scored as none, minimal, moderate or extensive);

Setting: developed versus less developed countries; health care setting; site of access (central location versus PDAs etc).

\section{Ongoing Studies}

On-going studies identified will be described, where available, detailing the primary author, research question(s), methods and outcome measures together with an estimate of the reporting date. 


\section{R E F E R E N CE S}

\section{Additional references}

\section{CMA, 2002}

Canadian Medical Association. CMA 2002 Physician Resource Survey. http://www.cmaj.ca/cgi/content/full/167/5/521/DC1 accessed 01 October 2003.

\section{Dawes, 2003}

.Dawes M, Sampson Uchechukwu S. Knowledge management in clinical practice: a systematic review of information seeking behavior in physicians. International Journal of Medical Informatics 2003;71:9-15.

\section{Judd 2004}

Judd M. Examining the use of evidence-based resources among physiotherapists in Canada. [dissertation] Ottawa, ON: University of Ottawa. 2004

\section{Kerse, 2001}

Kerse N, Arroll B, Lloyd T, Young J, Ward J. Evidence databases, the Internet, and general practitioners: the New Zealand story. The New Zealand medical journal 2001;114(1127):89-91.

\section{Kirk}

Kirk SFL, Cade JE, Greenhalgh A. Dietitians and the Internet: dietitians embracing the new technology?. Journal of Human Nutrition and Dietetics 2001;14:477-84.

\section{Nursing standard}

2002 RCN Publishing Company Internet Survey. http:// www.nursing-standard.co.uk/advertising/adpics/ ns_co_uk_media.pdf accessed 01 October 2003.

\section{Nylenna}

Nylenna M, Aasland OG. Physicians' Internet activities and their perceived coping with the medical information. Medscape General Medicine 2000;2:1.

\section{Ramsey}

Ramsey CR, Matowe L, Grilli R, Grimshaw JM, Thomas RE. Interrupted time series designs in health technology assessment: lessons from two systematic review of behavior change strategies. Intl J of Technology Assessment in Health Care 2003;19(4):1-11.

\section{rsom}

Royal Society of Medicine. Survey of Fellows' interest in the Internet. http://www.medscape.com/viewarticle/408041 accessed 01 October 2003.

\section{Wyatt, 2002}

Wyatt JC, Liu JL. Basic concepts in medical informatics. Journal of Epidemiology and Community Health 2002;56(11):808-812.

\section{WHAT'S NEW}

\begin{tabular}{lll}
\hline Date & Event & Description \\
\hline 2 April 2008 & Amended & Converted to new review format. \\
\hline
\end{tabular}

\section{HISTORY}

Protocol first published: Issue 2, 2004

Review first published: Issue 3, 2009

\begin{tabular}{lll}
\hline Date & Event & Description \\
\hline 12 February 2004 & $\begin{array}{l}\text { New citation required and major } \\
\text { changes }\end{array}$ & Substantive amendment \\
\hline
\end{tabular}

\section{CONTRIBUTIONS OFAUTHORS}

Jessie McGowan drafted the protocol, and amended it with comments from all co-reviewers. Reviewers will screen studies, examine studies for eligibility, extract data and analyze the results. LM will guide the data analysis planning. PT, RG, MJ and MD will assist with interpretation of the results and clinical relevance. GW will guide the data analyses.

\section{DECLARATIONSOF INTEREST}

None known. 


\section{SOURCES OF SUPPORT}

\section{Internal sources}

- Institute of Population Health, University of Ottawa, Canada.

- Belgian Centre for Evidence-Based Medicine, Belgium.

- Department of Family Medicine, McGill University, Canada.

- Department of Family Medicine, Laval University, Canada.

\section{External sources}

- Canadian Institutes of Health Research, Post-doctoral Fellowship grant, Canada.

- Fonds de la recherche en santé du Québec, Canada.

\section{N DEX TERMS}

\section{Medical Subject Headings (MeSH)}

Evidence-Based Medicine [ ${ }^{\star}$ statistics \& numerical data]; Guideline Adherence [statistics \& numerical data]; Health Personnel [ ${ }^{\star}$ statistics \& numerical data]; Patient Care; Professional Practice [*standards]; Randomized Controlled Trials as Topic

\section{MeSH check words}

Humans 